\title{
Paper-Based Batteries as Conductivity Sensors for Single-Use Applications
}

\author{
Laura Ortega, Anna Llorella, Juan Pablo Esquivel, and Neus Sabaté* \\ Cite This: ACS Sens. 2020, 5, 1743-1749 \\ Read Online
}

ABSTRACT: We present a novel approach to measure ionic conductivity with a selfpowered strategy. In particular, we propose the use of a paper-based battery as a sensor. The battery sensor unit consists of two electrodes placed side-by-side and covered by a piece of hydrophilic paper strip. The electrodes are externally connected to a resistive element. The addition of the fluid to be sensed-which acts as the electrolyte-activates the battery, which generates an output voltage that is dependent on the conductivity of the liquid sample. The device, which is conceived as a single-use disposable sensor, has been tested with different synthetic and biological liquid samples. The battery sensor effectiveness has been assessed by comparing its performance with a commercial laboratory conductometer. The device opens new avenues for conductivity monitoring in small portable and wearable devices, as it simplifies the number of electronic components and the need of additional power sources.

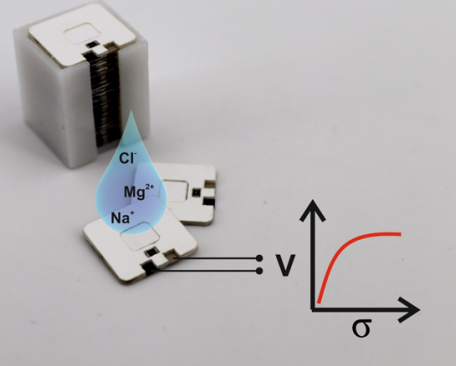

KEYWORDS: self-powered sensor, paper-based sensor, single-use, paper battery, conductivity

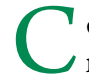
onductometry is a widely used method to perform the measurement of the electrolytic conductivity of a sample and determine its ionic content or to monitor the progress of a biochemical reaction. ${ }^{1}$ The electrochemical technique used to measure the conductivity of a liquid sample is the electrochemical impedance spectroscopy (EIS). EIS is a powerful electrochemical technique, which has the ability to study the intrinsic material property or specific processes that could influence the conductivity or capacity of an electrochemical system. ${ }^{2,3}$ EIS combines the analysis of both real and imaginary components of impedance, which are the conductivity of the electrolyte and the value of the impedance at high frequencies when the imaginary contribution is zero and only the real component is contributing. Specifically, to sense the conductivity of fluids, the most reported conductivity sensors are based on interdigitated electrode pairs, generally made of noble metal materials, to which a small sinusoidal alternating potential signal is applied, whereas the current induced between them is measured. The configuration of the electrode pairs and the distance between them defines the cell constant of the probe, impacting directly on the sensitivity of the device. ${ }^{4}$ Conductometry has proven to deliver relevant information in an extensive range of applications. For water resources, for example, conductivity is used to indicate whether the water is too saline to be drinkable or useable for irrigation. ${ }^{5,6}$ In the field of biosensing, conductivity sensors have been broadly applied to the study of enzymatic reactions that produce changes in the concentration of charged species as a result of the catalysis of a chemical compound (urea, Lasparagine, creatinine, etc.). ${ }^{2,7}$ They have also been used to directly monitor the changes in conductance of an electrode as a result of the immobilization of complementary antibodyantigen pairs, ${ }^{8,9}$ DNA, ${ }^{9}$ or whole cells. ${ }^{10,11}$

Whereas interdigitated electrodes for conductometry can be easily miniaturized and mass produced, ${ }^{12}$ an important limitation of the applicability of these sensors in portable and wearable devices is the electronic equipment associated with the mentioned systems that is needed, as to perform an impedance measurement the instrumentation required is complex and bulky in most cases. Moreover, as electrodes interact with the body fluids to monitor, operational time is limited and electrode reuse is generally avoided. For this reason, the biosensors tend to be incorporated in a single-use disposable platform/cartridge connected to a reusable electronic module. ${ }^{13-15}$ In recent years, the increase in personal health monitoring has led to devices where both single-use sensors and electronics are disposed of (e.g., the application does not require further monitoring-Clearblue pregnancy test-or the sensing consumable part is intimately integrated with the electronics and designed to be discarded at the same time-FreeStyle Libre glucose monitor from Abbott). This all-in-one trend is expected to grow significantly

Received: March 2, 2020

Accepted: May 20, 2020

Published: May 20, 2020 
a)

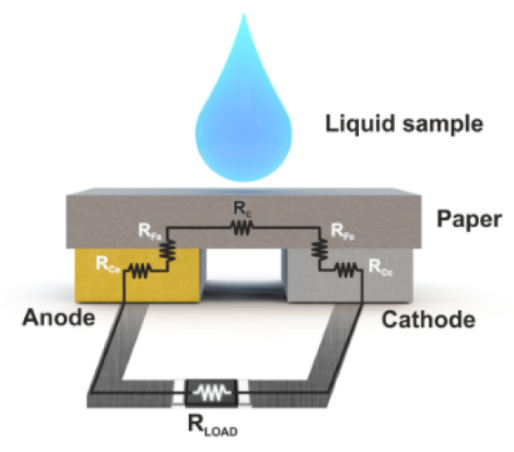

b)

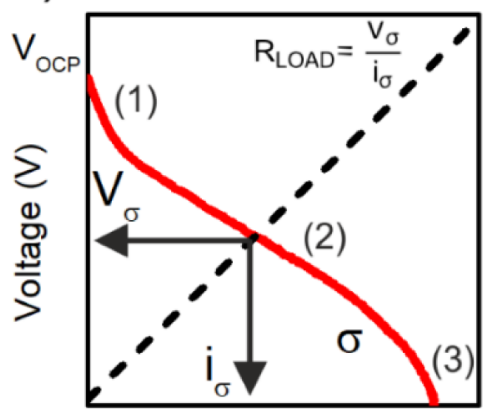

Current (A) c)

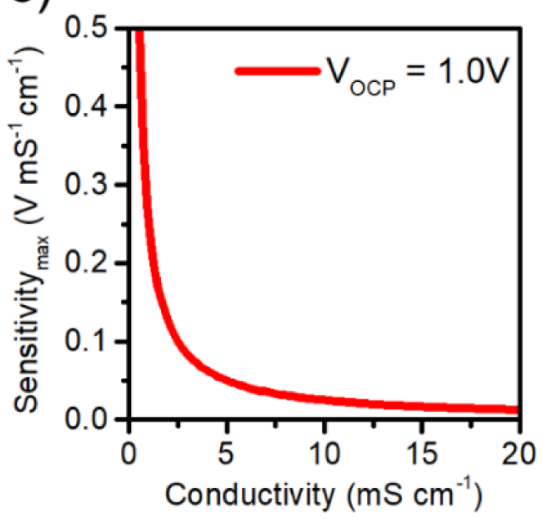

Figure 1. Basics of the conductivity paper battery sensor. (a) Scheme of the paper battery sensor showing all the resistive contributions governing the performance of the battery. (b) $I-V$ characteristic polarization curve of the paper battery (solid line). External resistive load $R_{\text {load }}$ connected to the battery (dashed line) intersects the $I-V$ curve in the Ohmic region so that the battery operates at $V_{\sigma}$ and $i_{\sigma}$, values directly related with the internal resistance of the paper battery sensor. (c) Maximum sensitivity obtainable with the paper battery sensor when $V_{\mathrm{OCP}}$ is 1 V.

in the upcoming wave of digital and personalized diagnostics. In this sense, self-powered galvanic sensors have arisen as a smart approach to ease and simplify single-use point-of-care devices, as they merge power sources and sensors into a single element and eliminate the need of additional power sources.

Remarkable examples of this are the lactate or the glucose single-use sweat patches ${ }^{16-20}$ that make use of the lactate and glucose anodic oxidation to build up a fuel cell able to generate power in proportion to the amount of analyte content.

In this paper, we present a novel approach that allows to measure ionic conductivity with a self-powered strategy. In particular, we propose to use a paper-based battery to sense ionic conductivity by using the liquid to be sensed as a battery electrolyte. The self-powered conductivity sensor effectivity to discriminate sweat conductivity when integrated in a single-use printed patch was recently reported. ${ }^{20}$

In this paper, we describe the operating principle of the paper battery as a stand-alone disposable sensor and show its suitability to measure different biological liquid samples in a very simple and affordable way.

\section{EXPERIMENTAL SECTION}

Chemicals and Samples. Solutions of $\mathrm{NaCl}$ (Sigma-Aldrich, St. Louis, Missouri, USA) were daily prepared and the conductivity and temperature of the solutions were measured with the $914 \mathrm{pH} /$ conductometer (Metrohm AG, Herisau, Switzerland). Artificial eccrine perspiration and artificial urine (Pickering Laboratories, Mountain View, CA, USA), phosphate buffer (Sigma-Aldrich, St. Louis, Missouri, USA), milk samples (Central Lechera Asturiana, Asturias, Spain), and pineapple juice samples (Conserve Italia Soc Coop Agricola, Bologna, Italy) were used to perform the final validation of the device.

Battery Fabrication. The battery was designed with CorelDraw (Corel, Ottawa, ON, Canada) and fabricated by rapid prototyping such as laser cutting (Mini 24, Epilog Laser, Golden, CO, USA). The device was mounted with pressure-sensitive adhesives of medical grade (Adhesive Research, Glen Rock, PA, USA) by stacking all the layers. The paper used to absorb the sample was a LF1 glass fiber filter from GE (General Electric, Boston, MA, USA). For the electrodes, the Mg anode was obtained from Galliumsource LLC (Scotts Valley, $\mathrm{CA}, \mathrm{USA}$ ) and the $\mathrm{AgCl}$ cathode was obtained by screen-printing with a manual screen-printer (PAYMSER, Barcelona, Spain). The screenprinting process of the cathode began with the collector track as the first layer and then the working electrode area was defined with the same technique. The track was printed with the Ag ink LOCTITE
ECI $1011 \mathrm{E} \& \mathrm{C}$ (Henkel, Düsseldorf, Germany) and cured at $150{ }^{\circ} \mathrm{C}$ for $15 \mathrm{~min}$. The working electrode was screen-printed over the track with the $\mathrm{Ag} / \mathrm{AgCl}$ ink C2140310D1 (Gwent Electronic Materials Ltd., Pontypool, Wales) and cured at $60{ }^{\circ} \mathrm{C}$ for $30 \mathrm{~min}$.

Electrochemical Characterization. The electrochemical characterization of the battery was performed with an Autolab potentiostat PGSTAT204 (Metrohm AG, Herisau, Switzerland).

Transient Measurements. The transient measurements to sense the output voltage of the battery once the resistor was connected in series were performed with an HP34401A multimeter (HewlettPackard Palo Alto, CA, USA) and controlled with a custom-made LabVIEW program (National Instruments, N Mopac Expy, Austin, TX, USA).

\section{RESULTS AND DISCUSSION}

Sensing Principle of the Paper Battery Sensor. The battery sensor unit-schematically depicted in Figure 1aconsists of two electrodes, the anode and the cathode, placed side-by-side and covered by a piece of hydrophilic paper strip. The electrodes are externally connected to a resistive element $\left(R_{\text {load }}\right)$. After the addition of a fluid onto the paper-which acts as an electrolyte-the battery is ready to function.

Under operation, the electrodes undergo a process of oxidation and reduction respectively that generates electric current. As it is well reported in the literature, the battery operating performance can be described from its polarization curve. $^{21}$

Polarization curves provide information about the voltage generated by the cell depending on the current demand. Figure $1 \mathrm{~b}$ depicts a scheme of a generic polarization curve. Departing from its open circuit potential $\left(V_{\mathrm{OCP}}\right)$ that depends on the battery chemistry, batteries experience voltage losses that are attributed to different phenomena: (1) activation losses because of the activation of the electrodes, (2) Ohmic losses because of ionic and electronic conduction across the battery, and (3) concentration losses because of the exhaustion of the electrodes. In the Ohmic loss region, the performance of the battery is directly related to the magnitude of its internal resistance, which is composed by the faradic resistance of the electrodes $\left(R_{\mathrm{F}}\right)$, the ionic resistivity of the electrolyte $\left(R_{\mathrm{E}}\right)$, and Ohmic resistance of the electrodes and the electrical contacts of the battery $\left(R_{\mathrm{C}}\right)$. Whereas $R_{\mathrm{F}}$ has a significant effect on voltage losses at low current ranges and becomes practically negligible at moderate currents, ionic and electronic 
resistivities are constant along the whole operating range of the battery. In particular, the ionic resistivity of the electrolyte depends on the geometrical dimensions of the paper, its porosity, and the ionic conductivity of the fluid used to activate its operation. Therefore, if the battery features are kept unaltered (component materials and geometry), the internal resistivity of the battery will change upon variations in the conductivity of the fluid, which will impact the slope of the Ohmic region of its polarization curve. This feature can be exploited to develop a simple yet smart, single-use, and selfpowered conductivity sensor.

One simple way to operate the battery sensor is to connect a resistor $\left(R_{\text {load }}\right)$ in series to the battery electrodes. Under this resistive load, the voltage output of the battery $\left(V_{\sigma}\right)$ will correspond to the value set by the intersection of the polarization curve at a given activation liquid conductivity and the loading resistor, as shown in Figure $1 \mathrm{~b}$. The output voltage of the battery can be derived from eqs 1 and 2, where $V_{\text {OCP }}$ corresponds to the open circuit voltage of the battery, $R_{\sigma}$ to the internal resistance of the battery at a given liquid electrolyte conductivity $(\sigma)$, and $i_{\sigma}$ and $V_{\sigma}$ correspond to the current and voltage output of the battery when connected to the external resistor.

$$
\begin{aligned}
& V_{\sigma}=V_{\mathrm{OCP}}-i_{\sigma} R_{\sigma}=i_{\sigma} R_{\text {load }} \\
& V_{\sigma}=\frac{V_{\mathrm{OCP}} \cdot R_{\mathrm{load}}}{R_{\sigma}+R_{\mathrm{load}}}
\end{aligned}
$$

It can be seen that according to (2), the voltage delivered by the battery depends on the value of its internal resistance, whereas the value of the external resistance connected to it modulates the battery response. In order to find out which is the value of $R_{\text {load }}$ that provides the maximum sensitivity to internal resistivity changes, we calculated the sensitivity (eq 3 ).

$$
S=\frac{\partial V_{\sigma}}{\partial R_{\sigma}} \frac{\partial R_{\sigma}}{\partial \sigma}=\frac{-V_{\mathrm{OCP}} R_{\text {load }}}{\left(R_{\sigma}+R_{\text {load }}\right)^{2}} \frac{\partial R_{\sigma}}{\partial \sigma}
$$

From this expression, it can be seen that the battery sensitivity to conductivity changes of the electrolyte diminishes at increasing conductivity values.

Moreover, it is easy to derive that the maximum sensitivity to internal resistance changes takes place when $R_{\text {load }}$ equals $R_{\sigma}$, that is, when the external resistance equals the value of the internal resistance of the battery. This means that selecting the loading resistor is a key step in the implementation of the conductivity battery sensor device, as for a particular conductivity range of interest, the maximum attainable sensitivity will be equal to

$$
S_{\max }=\left|\frac{V_{\mathrm{OCP}}}{4 R_{\sigma}} \frac{\partial R_{\sigma}}{\partial \sigma}\right|
$$

where $R_{\sigma}$ is a particular value of the internal resistivity of the battery previously selected.

In the case where Ohmic losses attributed to electrical contacts are minimized and the internal resistivity of the battery is dominated by the paper electrolyte resistivity, the internal resistance can be expressed as

$$
R_{\sigma}=R_{\text {electrolyte }}=\frac{k}{\sigma}
$$

where $k$ corresponds to the cell constant and $\sigma$ corresponds to the effective liquid conductivity, which is the conductivity of the liquid embedded in the paper matrix. In this case, the maximum sensitivity is independent of the cell geometry and can be directly related to the conductivity of the electrolyte.

$$
S_{\max }=\frac{V_{\mathrm{OCP}}}{4 \sigma}
$$

As an example, Figure 1c shows the maximal sensitivity values that would be obtained in a battery yielding $1 \mathrm{~V}$ of open circuit potential and neglecting the paper contribution to the electrolyte conductivity.

Paper Battery Sensor Description, Calibration, and Validation under Different Conductivity Samples. The battery sensor explored in this paper consists of a magnesium anode and a silver/silver chloride cathode of dimensions $2.5 \times$ $5 \mathrm{~mm}^{2}$ located side by side on a pressure-sensitive adhesive layer at a distance of $1.5 \mathrm{~mm}$. The electrodes are covered and connected with two layers of glass fiber-based paper with a total thickness of $0.5 \mathrm{~mm}$ and an area of $60 \mathrm{~mm}^{2}$. An exploded view of the device with all the different layers used in its fabrication is shown in Figure $\mathrm{S} 1$ of the Supporting Information. The glass-fiber paper is a highly hydrophilic material, which allows holding a liquid volume of $15 \mu \mathrm{L}$. Battery operation starts once the paper is completely filled with the liquid to be characterized. The basic electrochemistry of this battery has been used in the past in the so-called seawateractivated batteries. ${ }^{22,23}$ The reactions involved in the paper battery are as follows

$$
\begin{aligned}
& \text { anode: } \operatorname{Mg}(\mathrm{s}) \rightarrow \mathrm{Mg}^{2+}(\mathrm{aq})+2 \mathrm{e}^{-} \\
& \text {cathode: } \operatorname{AgCl}(\mathrm{s})+\mathrm{e}^{-} \rightarrow \mathrm{Ag}(\mathrm{s})+\mathrm{Cl}^{-}(\mathrm{aq}) \\
& \text { total: } \mathrm{Mg}(\mathrm{s})+2 \operatorname{AgCl}(\mathrm{s}) \\
& \quad \rightarrow \mathrm{Mg}^{2+}(\mathrm{aq})+2 \mathrm{Cl}^{-}(\mathrm{aq})+2 \mathrm{Ag}(\mathrm{s})
\end{aligned}
$$

The open-circuit voltage $\left(V_{\mathrm{OCP}}\right)$ in neutral media of $\mathrm{Mg}-$ $\mathrm{Ag} / \mathrm{AgCl}$ batteries is set at around $1.5-1.7 \mathrm{~V}^{24}$ In order to calibrate the performance of our battery at different ionic conductivities, we recorded the battery polarization curves when filled with water-based solutions containing different ionic conductivities.

Solutions with different $\mathrm{NaCl}$ concentrations were prepared to set liquid conductivities to the ranges typically found in body fluids and beverages $\left(0.5-10 \mathrm{mS} \mathrm{cm}^{-1}\right)$. Figure 2 a shows the obtained polarization curves of the battery once the solution was introduced in the paper matrix; then the open circuit voltage of the batteries was measured for $20 \mathrm{~s}$ and afterward a linear sweep voltammetry at $10 \mathrm{mV} \mathrm{s}^{-1}$ was performed from the open circuit voltage to $0 \mathrm{~V}$.

The effect of the ionic conductivity of the activation liquid in the Ohmic region of the curves is clear. It can be also noticed that voltage losses related to the faradaic resistance of the electrodes in the activation zone of the $I-V$ curve are significant, in particular at conductivity ranges below $3 \mathrm{mS}$ $\mathrm{cm}^{-1}$. An individual electrochemical characterization of both magnesium and silver/silver chloride electrodes was performed to analyze the origin of the activation losses observed in Figure $2 \mathrm{a}$. The half-cell measurements with both electrodes were performed with a commercial $\mathrm{Ag} / \mathrm{AgCl}$ as the reference electrode and a Pt electrode as the counter electrode inside a beaker. The magnesium anode and the silver/silver chloride cathode were connected as the working electrode in each of the experiments. A linear sweep voltammetry at $10 \mathrm{mV} \mathrm{s}^{-1}$ at 

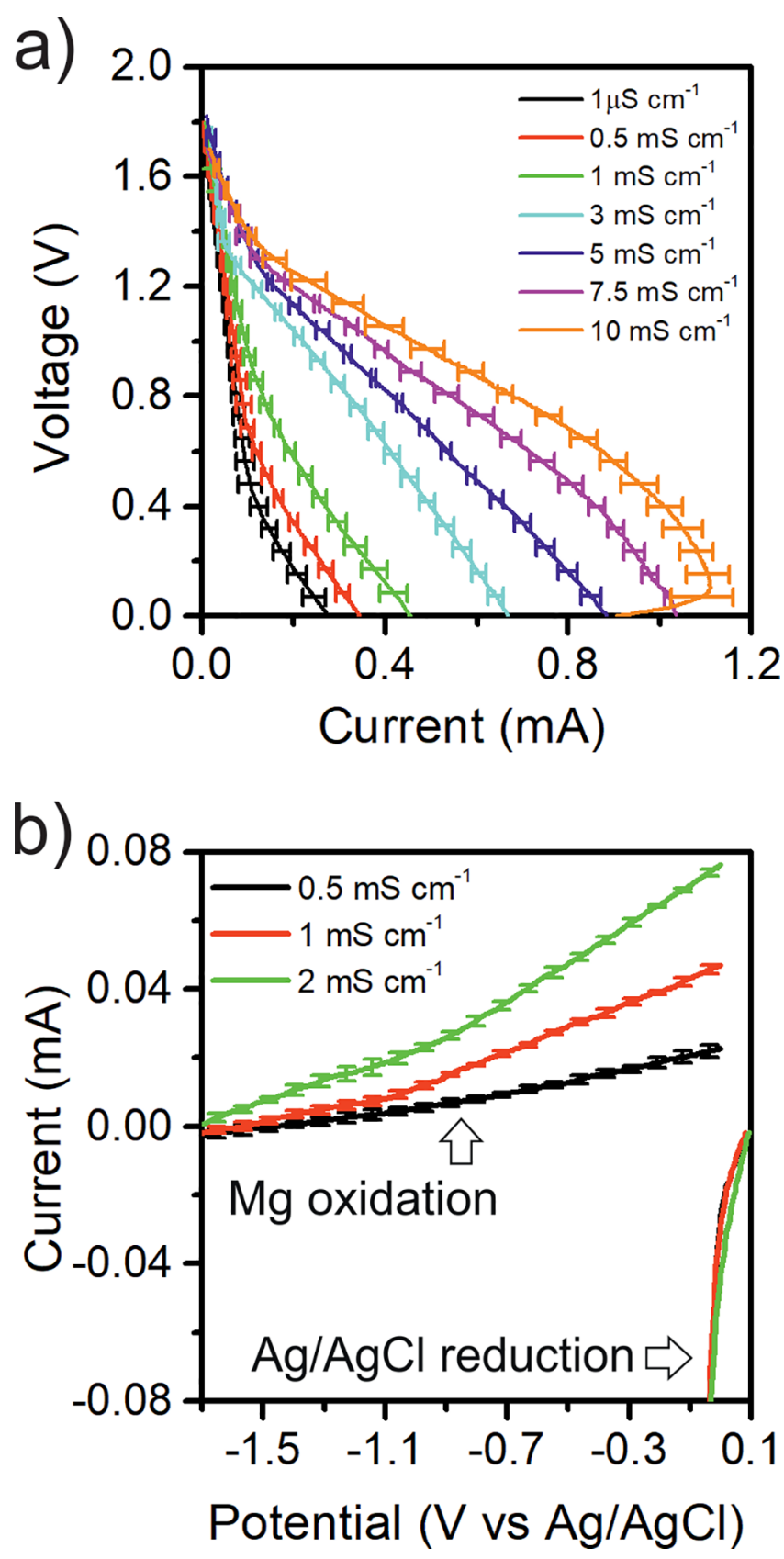

Figure 2. Characterization of the fabricated conductivity paper battery sensor. (a) Polarization curves of the paper battery sensor when tested at conductivities from $1 \mu \mathrm{S} \mathrm{cm}^{-1}$ to $10 \mathrm{mS} \mathrm{cm}^{-1}$. (b) Partial reactions of the paper battery sensor electrodes when introducing three different electrolyte conductivities. It shows the activation losses of the anode and the nonrestrictive performance of the cathode.

different conductivities with the two electrodes revealed that the activation losses were dominated by the magnesium anode, as shown in Figure 2b, probably because of the existence of a passivating layer of magnesium oxide in the anode. The effect of this passivating layer becomes less prominent at increasing conductivities, mainly because the presence of chloride ions increases the rate of the anodic dissolution kinetics. ${ }^{25}$

To use this battery as a self-powered sensor that allows deriving information of the conductivity of a liquid sample, a resistor is connected in series to the battery electrodes. As it has been previously mentioned, the value of this resistor has to be selected so it places the battery operation within its Ohmic region for the whole conductivity range to be measured. As it can be observed in Figure 3a, for this specific battery geometry and composition, suitable values of $R_{\text {load }}$ range from 1 to 2.5 $\mathrm{k} \Omega$. In fact, these values correspond to the values of internal resistance measured in the battery sensor at the lower and upper limits of the tested conductivity range. Figure $3 \mathrm{~b}$ shows the battery sensor output voltage derived from the intersection of $R_{\text {load }}$ with the battery polarization curves for resistor values of 1 and $2.5 \mathrm{k} \Omega$. They also define the voltage operating window of the cell. As expected, higher output voltages are obtained at higher $R_{\text {load }}$ values because the battery operates at the upper zone of its Ohmic region. The derivative of the voltage curves allowed us to obtain the sensitivity of the sensor along the whole tested conductivity range, Figure $3 \mathrm{c}$. It can be seen that a higher sensitivity is achieved at a higher value of resistive load and that, as expected, sensitivity decreases significantly at increasing conductivity values. At the same time, the impact of $R_{\text {load }}$ on sensitivity becomes less evident at conductivities above $5 \mathrm{mS} \mathrm{cm}^{-1}$. Sensitivity values at low conductivities depart from the ideal $1 / \sigma$ dependence because of the effect of the pre-existing passivation layer at the magnesium electrodes.

Once the paper battery performance was calibrated within the conductivity range of interest, a battery sensor consumable was fabricated by incorporating a resistor in the paper-based device (see Figure 4a). In order to obtain the highest sensitivity response, a value of $2.5 \mathrm{k} \Omega$ was selected. The assembly was then thoroughly calibrated under continuous operation with different $\mathrm{NaCl}$ solutions. The battery was activated with $15 \mu \mathrm{L}$ of the liquid samples and left to stabilize for $5 \mathrm{~s}$. After that, the battery output voltage was recorded for 1 $\min$. Figure $4 \mathrm{~b}$ shows the voltages generated by the battery over time at different conductivity values. It can be seen that battery voltages showed a significant stability for conductivities over $1.0 \mathrm{mS} \mathrm{cm} \mathrm{cm}^{-1}$ along the measurement period, whereas for lower conductivity values $\left(0.5\right.$ and $\left.1.0 \mathrm{mS} \mathrm{cm}^{-1}\right)$, the battery voltage showed a slight increase over time. This small voltage variation is due to the progressive removal of the passivation layer. During the calibration, the voltage output of the battery presented a repetitive behavior, providing voltages with a coefficient of variation of less than $10 \%$ in all cases. A calibration curve was obtained by depicting the battery voltage output at a particular time $(30 \mathrm{~s})$ as shown in Figure $4 \mathrm{c}$. The resulting values were fitted to expression (2) to which an additional term $\left(V_{0}\right)$ had been added to account for the experimental offset value provided by the battery when activated with deionized water $\left(1 \mu \mathrm{S} \mathrm{cm}^{-1}\right)$, obtaining eq 7 . Substituting the constant parameters in (7), the experimental results were fitted to the nonlinear final expression (8), where $k$ corresponds to the cell constant and $V_{\mathrm{OCP}}^{*}$ to an effective value of the open circuit potential of the battery (to which activation losses have been subtracted).

A $V_{0}$ value of $0.33 \mathrm{~V}$ was extracted from the intersection of the corresponding polarization curve shown in Figure 2a with a $R_{\text {load }}$ of $2.5 \mathrm{k} \Omega$. Fitting of the experimental values yielded an adjusted $R$-square of 0.9943 and $k$ and $V_{O C P}^{*}$ coefficients that are presented in Table 1.

$$
V_{\text {out }}=\frac{V_{\mathrm{OCP}}^{*} \cdot R_{\text {load }}}{\frac{k}{\sigma}+R_{\text {load }}}+V_{0}
$$



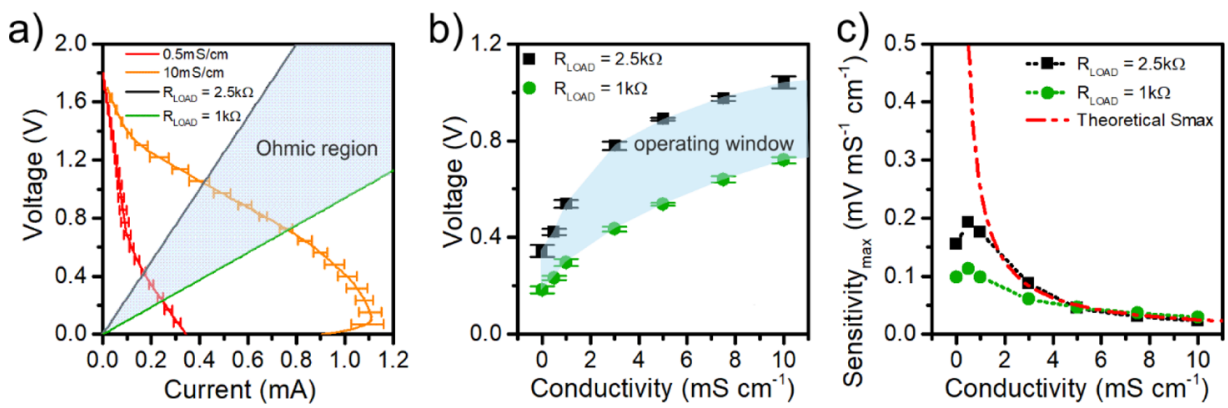

Figure 3. Analysis of the conductivity paper battery sensor performance. (a) Polarization curves of the paper battery sensor at the lower and upper values of the tested conductivity range $\left(0.5\right.$ and $\left.10 \mathrm{mS} \mathrm{cm}^{-1}\right) . R_{\text {load }}$ from 1 to $2.5 \mathrm{k} \Omega$ (green and black solid lines) set the battery into the Ohmic region operation. (b) Output voltages obtained from the intersection of the battery polarization curves with $R_{\text {load }}$ set to $1 \mathrm{k} \Omega$ (green dots) and 2.5 $\mathrm{k} \Omega$ (black squares). (c) Sensitivity values of the battery obtained from the experimental polarization curves vs the theoretical maximum sensitivity (dashed red line).

a)

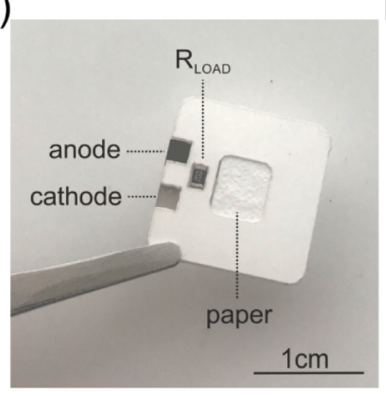

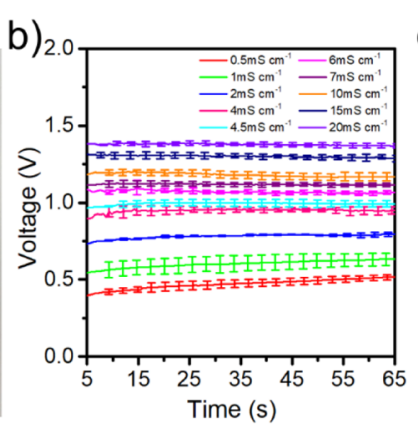

c)

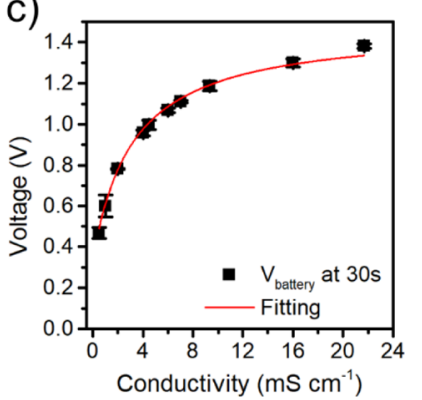

Figure 4. (a) Picture of a ready-to-use conductivity battery sensor. (b) Battery output voltages under continuous operation at conductivities from 0.5 to $20 \mathrm{mS} \mathrm{cm}^{-1}$. (c) Calibration curve of the battery sensor.

$$
V_{\text {out }}=\frac{V_{\mathrm{OCP}}^{*} \cdot 2.5}{\frac{k}{\sigma}+2.5}+0.33
$$

Table 1. Battery Voltage Output Fitting Parameters

\begin{tabular}{clc}
\hline parameter & value & standard error \\
\hline$k$ & 7.8 & 0.3 \\
$V_{\mathrm{OCP}}^{*}$ & 1.15 & 0.02 \\
\hline
\end{tabular}

After the battery sensor calibration, the device performance as a single-use self-powered conductometer was validated by measuring conductivities of real liquid samples such as milk, juice, artificial eccrine perspiration, urine, lake water, and phosphate butter. Figure 5a shows the battery output voltages recorded for the different samples versus time with the same methodology used for the calibration. As it can be seen, the battery sensor delivered output voltages with a coefficient of variations of less than $10 \%$ in all cases regardless of the origin, synthetic or natural. Again, the voltage output of the battery recorded at $30 \mathrm{~s}$ was used in expression (8) to derive the conductivity of the samples.

It is worth noticing that as the sensor is a primary battery, its operational time depends on its charge capacity and the current intensity at which this charge is being released. In the present case, the $\mathrm{Ag} / \mathrm{AgCl}$ electrode was significantly thinner than the $\mathrm{Mg}$ anode and therefore, the total charge capacity of the sensor battery was limited by the total quantity of $\mathrm{AgCl}$ molecules available for reduction. Measurements of the current delivered by our sensor batteries under three different electrolyte conductivities until electrode exhaustion yielded an average charge capacity of $45 \mathrm{mC}$, which set to $80 \mathrm{~s}$ the maximum operational time at the highest conductivity tested value $\left(20 \mathrm{mS} \mathrm{cm}^{-1}\right)$. Details of experiments and calculations are provided in Figure S2.

Finally, the conductivity values measured by the paper battery sensor were compared with those provided with a commercial lab conductometer, $914 \mathrm{pH} /$ conductometer (Metrohm). Figure $5 \mathrm{~b}$ shows the Passing-Bablok linear regression comparing the results provided by the paper battery sensor device against the lab conductometer results. It can be seen that the conductivity values obtained with the paper battery sensor show a good correlation with the values obtained with the reference method except in the case of phosphate buffer. A table containing the conductivity values recorded with the reference method and the ones obtained with the battery sensor can be found in Table S1. The discrepancy is probably due to the interaction of phosphate salts with the magnesium ions released from the anode. $\mathrm{Mg}^{2+}$ has a high affinity for many anionic salts. Although most of the magnesium salts have a relatively high solubility constant $\left(K_{\mathrm{ps}}\right)$ in aqueous media, there are a few that precipitate at very low concentrations. In particular, when mixed with carbonates, fluorides, hydroxides, sulfates, or phosphates, the resulting magnesium salts form precipitates. In this case, the solubility of $\mathrm{Mg}^{2+}$ and $\mathrm{PO}_{4}^{-}$corresponds to a $K_{\mathrm{ps}}$ of $4 \times 10^{-25}$, which reinforces the idea of electrode passivation by magnesium phosphate precipitates that is responsible for the observed deviation in the conductivity measurement of the buffer. The interaction of $\mathrm{Mg}^{2+}$ ions released by the anode with some specific anionic components restricts the applicability of the sensor to selected environments. In this sense, our sensor is particularly suited to measure conductivity biological samples 

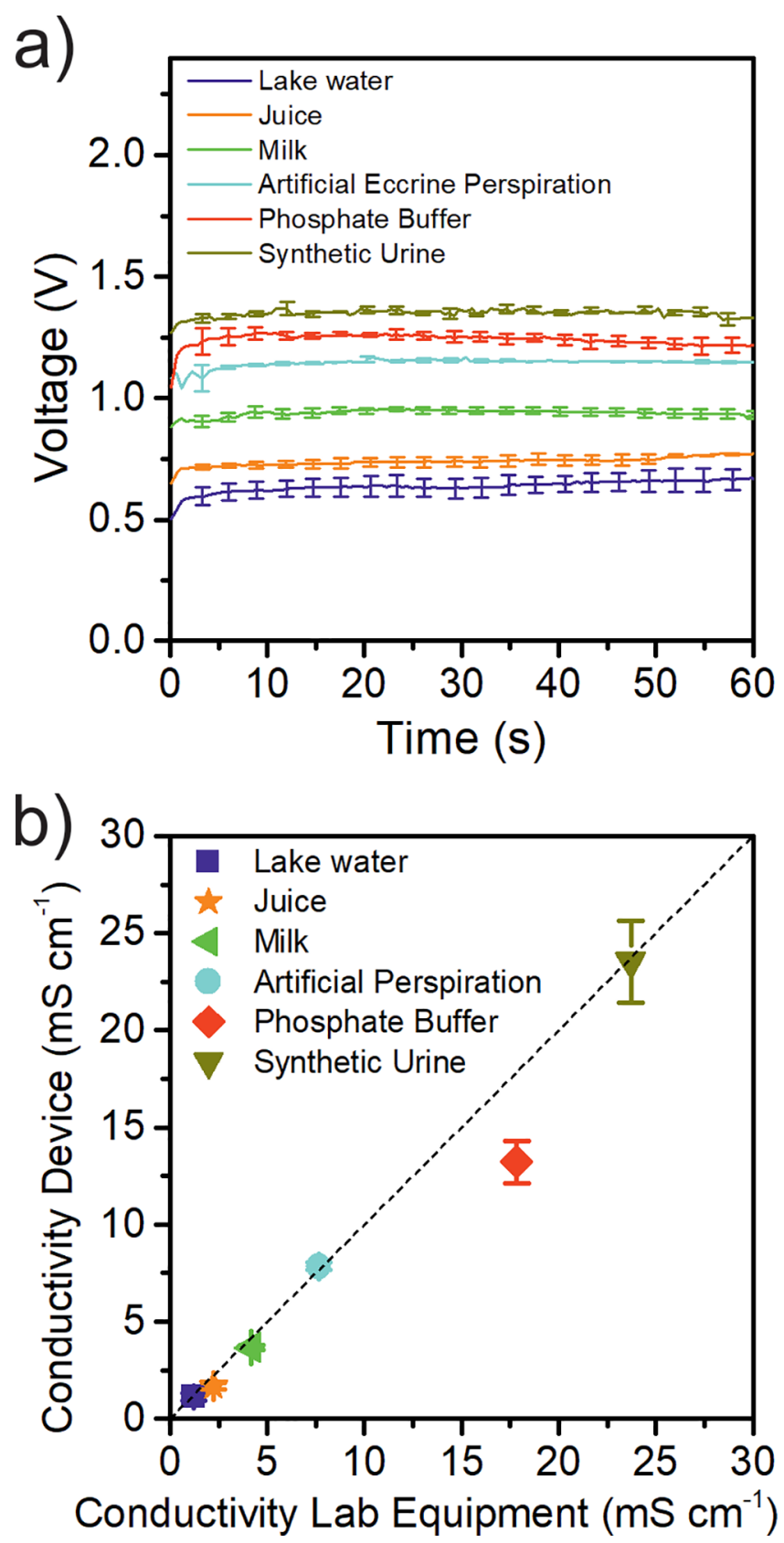

Figure 5. (a) Battery output voltages under continuous operation when measuring real samples. (b) Passing-Bablok linear regression that compares the conductivity values obtained with the paper battery sensor device against the lab reference method.

where these interfering salts are seldom present and, generally, at submillimolar concentrations. ${ }^{26,27}$ However, the interaction of the electrodes with ionic species present in the liquid sample can be further prevented by introducing ion-selective membranes as effective separators.

\section{CONCLUSIONS}

This paper presents a novel method to measure fluid conductivity that does not require complex instrumentation or costly setups. In particular, the use of a paper-based battery connected to a single resistor allows to obtain a dc output that is not only easy to interpret with a simple handheld multimeter but is also self-powered, as the battery acts as a sensor and a power source at the same time.
The availability of a battery whose output voltage becomes informative of the conductivity of a sample opens new avenues for self-powered sensing strategies and their application to portable and wearable devices. The connection of a resistor allows obtaining both power (voltage and current) and information about the sample, which is coded in the output voltage of the battery. There are many ways of translating output voltage to meaningful information about the conductivity of the sample by solely using the power generated by the battery sensor. We recently showed that a simple circuit consisting of a single transistor and a pair of resistors acting as a tunable voltage divider could enable a clinically relevant discrimination of cystic fibrosis occurrence in a single-use sweat patch. The sensor output information was delivered by two electrochromic displays that were powered by the battery sensor itself. $^{20}$ Departing from this simple but effective realization, the possibilities for development of smart circuits to quantify the battery voltage and develop other formats and applications that involve conductivity measurements are unlimited.

The self-powered strategy followed in this paperconnection of a resistor in parallel to the battery-limits the dynamic range of the sensor. As sensitivity decreases at a pace of $1 / \sigma$, there is an unavoidable sensor saturation at increasing conductivities. However, there is room to explore new engineering strategies (i.e., operating the cell at a fixed voltage in a self-powered mode or connecting a battery stack to obtain multiples of open circuit potential $\left.\left(V_{\mathrm{OCP}}\right)\right)$ that will allow expanding the sensor dynamic range beyond the values presented in this paper.

Furthermore, the device presented here requires a very small volume to perform the measurement $(15 \mu \mathrm{L})$, which is significantly smaller than the milliliter range volumes required by common conductivity probes. This turns out to be especially suitable for conductivity measurements of biological samples such as blood, tears, or sweat, in which availability of sample volume is very limited. Although theoretically possible, the device cannot be reused in practice. The electrodes undergo irreversible redox reactions that modify their surface and the cell characteristics may be altered from one measurement to the next. Because of its nature, the device has been considered to be single-use. However, the simplicity of both materials used and fabrication processes required to develop the sensor allow to envisage its rapid adoption by the booming generation of single-use and paper-based analytical devices whenever conductivity measurements are needed.

\section{ASSOCIATED CONTENT}

\section{Supporting Information}

The Supporting Information is available free of charge at https://pubs.acs.org/doi/10.1021/acssensors.0c00405.

Exploded vision of the paper battery sensor, relative errors observed when comparing the conductivity values obtained by the paper battery sensor, and lab conductometer and lifetime of the battery sensor under different conductivity regimes (PDF)

\section{AUTHOR INFORMATION}

\section{Corresponding Author}

Neus Sabaté - Instituto de Microelectrónica de Barcelona, IMBCNM (CSIC), 08193 Barcelona, Spain; Catalan Institution for Research and Advanced Studies (ICREA), 08010 Barcelona, 
Spain; ๑ orcid.org/0000-0002-4513-7057;

Email: neus.sabate@imb-cnm.csic.es

\section{Authors}

Laura Ortega - Instituto de Microelectrónica de Barcelona, IMB-CNM (CSIC), 08193 Barcelona, Spain

Anna Llorella - Instituto de Microelectrónica de Barcelona, IMB-CNM (CSIC), 08193 Barcelona, Spain

Juan Pablo Esquivel - Instituto de Microelectrónica de Barcelona, IMB-CNM (CSIC), 08193 Barcelona, Spain

Complete contact information is available at:

https://pubs.acs.org/10.1021/acssensors.0c00405

\section{Author Contributions}

All the authors conceived the device concept. J.P.E. assessed the prototype design. L.O. and A.L. carried out fabrication and characterization of the battery sensor with direction from N.S. All the authors contributed to the paper preparation.

\section{Notes}

The authors declare no competing financial interest.

\section{ACKNOWLEDGMENTS}

N.S. would like to thank the financial support received from the ERC Consolidator grant (SUPERCELL-GA.648518).

\section{REFERENCES}

(1) Khopkar, S. M. Basic Concepts of Analytical Chemistry, 3rd ed.; New Academic Science: Tunbridge Wells, 2012.

(2) Cullen, D. C.; Sethi, R. S.; Lowe, C. R. Multi-Analyte Miniature Conductance Biosensor. Anal. Chim. Acta 1990, 231, 33-40.

(3) Grieshaber, D.; MacKenzie, R.; Vörös, J.; Reimhult, E. Electrochemical Biosensors - Sensor Principles and Architectures. Sensors 2008, 8, 1400-1458.

(4) Olthuis, W.; Streekstra, W.; Bergveld, P. Theoretical and Experimental Determination of Cell Constants of Planar-Interdigitated Electrolyte Conductivity Sensors. Sensor. Actuator. B Chem. 1995, 24, 252-256.

(5) Allen, R. G.; Pereira, L. S.; Raes, D.; Smith, M. Crop Evapotranspiration-Guidelines for Computing Crop Water Requirements-FAO Irrigation and Drainage Paper 56; Food and Agriculture Organization: Rome, 1998; Vol. 300, 9.

(6) European Commission. Council Directive 98/83/EC on the Quality of Water Intended for Human Consumption; Official Journal of the European Communities, 1998.

(7) Chin, W.-t.; Kroontje, W. Conductivity Method for Determination of Urea. Anal. Chem. 1961, 33, 1757-1760.

(8) Pei, R.; Cheng, Z.; Wang, E.; Yang, X. Amplification of AntigenAntibody Interactions Based on Biotin Labeled Protein-Streptavidin Network Complex Using Impedance Spectroscopy. Biosens. Bioelectron. 2001, 16, 355-361.

(9) Katz, E.; Willner, I. Probing Biomolecular Interactions at Conductive and Semiconductive Surfaces by Impedance Spectroscopy: Routes to Impedimetric Immunosensors, DNA-Sensors, and Enzyme Biosensors. Electroanalysis 2003, 15, 913-947.

(10) Lei, Y.; Chen, W.; Mulchandani, A. Microbial Biosensors. Analytica Chimica Acta 2006, 568, 200-210.

(11) Brosel-Oliu, S.; Mergel, O.; Uria, N.; Abramova, N.; Van Rijn, P.; Bratov, A. 3D Impedimetric Sensors as a Tool for Monitoring Bacterial Response to Antibiotics. Lab Chip 2019, 19, 1436-1447.

(12) Jaffrezic-Renault, N.; Dzyadevych, S. Conductometric Microbiosensors for Environmental Monitoring. Sensors 2008, 8, 25692588.

(13) Emaminejad, S.; Gao, W.; Wu, E.; Davies, Z. A.; Yin Yin Nyein, H.; Challa, S.; Ryan, S. P.; Fahad, H. M.; Chen, K.; Shahpar, Z.; Talebi, S.; Milla, C.; Javey, A.; Davis, R. W. Autonomous Sweat Extraction and Analysis Applied to Cystic Fibrosis and Glucose
Monitoring Using a Fully Integrated Wearable Platform. Proc. Natl. Acad. Sci. U.S.A. 2017, 114, 4625-4630.

(14) Montes-Cebrián, Y.; del Torno-de Román, L.; Álvarez-Carulla, A.; Colomer-Farrarons, J.; Minteer, S. D.; Sabaté, N.; Miribel-Català, P. L.; Esquivel, J. P. 'Plug-and-Power' Point-of-Care Diagnostics: A Novel Approach for Self-Powered Electronic Reader-Based Portable Analytical Devices. Biosens. Bioelectron. 2018, 118, 88-96.

(15) Nyein, H. Y. Y.; Bariya, M.; Kivimäki, L.; Uusitalo, S.; Liaw, T. S.; Jansson, E.; Ahn, C. H.; Hangasky, J. A.; Zhao, J.; Lin, Y.; Happonen, T.; Chao, M.; Liedert, C.; Zhao, Y.; Tai, L. C.; Hiltunen, J.; Javey, A. Regional and Correlative Sweat Analysis Using HighThroughput Microfluidic Sensing Patches toward Decoding Sweat. Sci. Adv. 2019, 5, No. eaaw9906.

(16) Bandodkar, A. J.; You, J.-M.; Kim, N.-H.; Gu, Y.; Kumar, R.; Mohan, A. M. V.; Kurniawan, J.; Imani, S.; Nakagawa, T.; Parish, B.; Parthasarathy, M.; Mercier, P. P.; Xu, S.; Wang, J. Soft, Stretchable, High Power Density Electronic Skin-Based Biofuel Cells for Scavenging Energy from Human Sweat. Energy Environ. Sci. 2017, $10,1581-1589$.

(17) Valdés-Ramírez, G.; Li, Y.-C.; Kim, J.; Jia, W.; Bandodkar, A. J.; Nuñez-Flores, R.; Miller, P. R.; Wu, S.-Y.; Narayan, R.; Windmiller, J. R.; Polsky, R.; Wang, J. Microneedle-Based Self-Powered Glucose Sensor. Electrochem. commun. 2014, 47, 58-62.

(18) Imani, S.; Bandodkar, A. J.; Mohan, A. M. V.; Kumar, R.; Yu, S.; Wang, J.; Mercier, P. P. A Wearable Chemical-Electrophysiological Hybrid Biosensing System for Real-Time Health and Fitness Monitoring. Nat. Commun. 2016, 7, 11650.

(19) Cho, E.; Mohammadifar, M.; Choi, S. A Single-Use, SelfPowered, Paper-Based Sensor Patch for Detection of ExerciseInduced Hypoglycemia. Micromachines 2017, 8, 265.

(20) Ortega, L.; Llorella, A.; Esquivel, J. P.; Sabaté, N. Self-Powered Smart Patch for Sweat Conductivity Monitoring. Microsyst. Nanoeng. 2019, $5,3$.

(21) Linden, D.; Reddy, T. B. Handbook of Batteries; McGraw-Hill Handbooks; McGraw-Hill, 2002.

(22) Carson, W. N., Jr.; Fischer, W. H. Sea Water-Activated Primary Battery. U.S. Patent 3,352,718 A, July 23, 1963.

(23) Koontz, R. F.; Lucero, R. D. Magnesium Water-Activated Batteries. Handbook of Batteries; McGraw-Hill, 2002; pp 1-27, Chapter 17.

(24) Zhang, T.; Tao, Z.; Chen, J. Magnesium-Air Batteries: From Principle to Application. Materials Horizons; Royal Society of Chemistry, 2014; Vol. 1, pp 196-206.

(25) King, A. D.; Birbilis, N.; Scully, J. R. Accurate Electrochemical Measurement of Magnesium Corrosion Rates; A Combined Impedance, Mass-Loss and Hydrogen Collection Study. Electrochim. Acta 2014, 121, 394-406.

(26) Prompt, C. A.; Quinto, P. M.; Kleeman, C. R. High Concentrations of Sweat Calcium, Magnesium and Phosphate in Chronic Renal Failure. Nephron 1978, 20, 4-9.

(27) Cundy, T.; Reid, I. R.; Grey, A. Metabolic Bone Disease. Clinical Biochemistry: Metabolic and Clinical Aspects, 3rd ed.; Elsevier Inc., 2014; pp 604-635. 